Human Heredity 1971;21:634-636

\title{
Subject Index Vol. 21
}

A antigen titres in saliva, Australian populations 334

A blood group subtypes in Caucasians and Negroes 88

ABO blood groups in Burma 420

Chile 431

India 326

Acid phosphatase variants, see AP types

ADA-P blood group linkage 351

ADA types in Denmark 63

India 440

Israel 357

lymphocytes 607

North Germany 278

-, world distribution 61

Adenosine deaminase variants, see ADA types

Adenylate kinase variants, see AK types

Africa, South, see South Africa

Ag types in Greenland Eskimos 129, 384

Norwegian Lapps 134

paternity cases 493

AK types in Denmark 83

India 297, 440

Israel 289

Malaysia 173

North Germany 278

-, segregation 371

-, world distribution 302

Albumin-Gc linkage 248

Albumin variants in Canadian Indians 242

Greenland Eskimos 57

Indonesia 376

Malaysia 376

North India 440

Alkaline phosphatase levels in newborns

and their mothers 69 Alpha-thalassaemia in newborns 185 AP types in North Germany 278 India 440

-, segregation 371

Arm folding types in Manipuris (India) 203

, recording 509 
Atopy and juvenile diabetes mellitus 504 ATP-ase deficiency, familial 313 Australia, A, H and Le(a) antigen titres in

saliva 334

Beta-lipoproteín variation 614 Birth weight and chromosome abnormalities 543 Blood groups, Burmese population 420

India 326

see also ABO and Rh blood groups Brachmann-de Lange syndrome 1 Brazil, haemophilia 97

Burmese population, blood groups 420

Canadian Indians, see Indians Cardiovascular malformations, congenital, epidemiology 209

Subject Index

635

Cell cultures, G-6-PD and PGM types 238

- $\quad$ naphthylamidase types 580, 591

Chile (Valparaiso), ABO blood groups and

colour vision deficiency 431

Chinese, Gc, Gm and Inv types 168, 362

Chromosome abnormalities, effects on birth weight 543

trisomy 17-18 and 21 in siblings 577

Chromosome measurements in MZ twins 557

Cleft lip and palate, epidemiology (Hungary) 17

Colour vision deficiency in Valparaiso (Chile) 431

Congenital cardiovascular malformations, epidemiology 209

Cornelia de Lange syndrome 1

C 3 system, family study 458

C 3 types in Denmark 272

Norway 154

Norwegian Lapps 162

Sweden 263

Swedish Lapps 267

Denmark, C 3 types 272 Dermatoglyphics, a new syndrome 39 Dermatoglyphics in

Habbanite Isolate 478

India (Mysore) 192

Mexican Indians 394

Schizophrenia 198 -, see also Triradius t

Diabetes mellitus, juvenile, and atopy 504

Ectodermal dysplasias 309 Eskimos, see Greenland

Finland, PGM distribution 140 -, Lapps, see Lapps

Gc-albumin linkage 248

Gc types in Canadian Indians 242

Chinese 362

Norwegian Lapps 134

Germany, red cell enzyme polymorphisms

278

Gm types in Chinese 168, 362

Greenland Eskimos 405

Scotland 628 
Greenland Eskimos, Ag types 129, 384

albumin variants 57

Gm types 405

Lp types 129 -PGM types 147

G-6-PD deficiency in India 78 G-6-PD types in cultured cells 238

Habbanite Isolate, dermatoglyphics 478

Haemophilia in Brazil 97

Hand clasping in Manipuris (India) 203

$\mathrm{H}$ antigen titres in saliva, Australian populations 334

Heart malformations, congenital, epidemiology 209

Heterophile antibodies in South African populations 180

Hp types in Canadian Indians 242

North India 440

Hungary, epidemiology of cleft lip and palate 17

Inbreeding coefficient, Japan 216 Incestuous matings, children of 108 India, ABO and Rh

blood groups 326 -AK types 297

arm folding types 203

G-6-PD deficiency 78

hand clasping types 203

palmar dermatoglyphics 192

pseudocholinesterase variants 388

serum protein and enzyme groups 440 Indians, Canadian, serum protein polymorphisms 242

Mexico, dermatoglyphics 394 Indonesia, albumin variants 376 Inv types in Chinese 168, 362

Isochromosomes from C-group chromosome 231

Israel, ADA types 357

-, AK and PGM types 289

Japan, inbreeding coefficient 216

Lapps, Finland, PGM types 140

- $\quad$ Norway, C 3 types 162

- $\quad$ serum protein polymorphisms 134

636

Subject Index

- $\quad$ Sweden, C 3 types 267

$\mathrm{LDH}$ variants in India 440

Lewis(a) antigen titres in saliva, Australian

population 334 Linkage, albumin-Gc 248

relations of ADA locus 351

Lp types in Greenland Eskimos 129

Norwegian Lapps 134

Malaysia, AK variants 173

albumin variants 376

alpha-thalassaemia in newborns 185 Mental deficiency and sex chromatin 54 Methods, analysis of pedigree data 525 Mexican Indians, dermatoglyphics 394 MN blood groups, Burma 420 Mutation rate, haemophilia 103

NADH diaphorase variants (India) 440 Naphthylamidase variants in cultured cells 580, 591 
neoplastic cells 500

Negroes, A blood group subtypes 88 Neoplastic cells, naphthylamidase isozymes 500 Norway, C 3 types 154 -, Lapps, see Lapps -, placental PGM types 234 Nya blood group 346

Palmar dermatoglyphics, see Dermatoglyphics P blood group - ADA linkage 351 Pedigree data, genetic analysis 525 PGI variants 467 PGM types in cultured cells 238

Finns 140

Finnish Lapps 146

Greenland Eskimos 147

India 440

Israel 289

Northern Germany 278

placenta (Norway) 254

USSR 147

PGM types, segregation 371 Phosphoglucomutase, see PGM Phosphoglucose isomerase variants 467 Phosphohexose isomerase variants (India)

440 Placental PGM types (Norway) 254 Pseudocholinesterase variants in India

(Panjabis) 388

Rh blood groups in Burma 420

India 326

Schizophrenia, dermatoglyphics 198 -etiology 517

Scotland, Gm (1) allotypes 628 Selection against recessive alleles 320 Sex chromatin and mental deficiency 54 6-PGD types in North Germany 278

India 440

, world distribution 305

South African populations, heterophile

antibody levels 180

Sweden, C 3 types 263

in Lapps 267

Tf types in Canadian Indians 242

India 440

Norwegian Lapps 134

Triradius t, methods of location 624 Trisomy 17-18 and 21 in siblings 577 Twins, MZ, chromosome measurements 557

USSR, northwestern, PGM distribution 147 